\title{
Assessment of Cancer-Related Dysfunctional Beliefs about Sleep for Evaluating Sleep Disturbance in Cancer Patients
}

\author{
Seockhoon Chung, MD, PhD, Soyoung Youn, MD, Byeongil Choi, MD \\ Department of Psychiatry, Asan Medical Center, University of Ulsan College of Medicine, Seoul, Korea
}

\begin{abstract}
Dysfunctional beliefs about sleep may be associated with chronic sleep disturbance in patients afflicted with cancer over the trajectory of their battle with cancer. The objective of this study was exploration of the utility of assessing the sleep disturbances suffered by cancer patients possibly emanating from two cancer-related informationally valid but nonetheless dysfunctional beliefs: "My immune system will have serious problems if I don't go to sleep at a certain time (question 1)" and "If I don't sleep well at night, my cancer can recur or metastasize (question 2)." The scores for these beliefs were significantly correlated with insomnia severity [measured using the Insomnia Severity In$\operatorname{dex}(\mathrm{p}<0.01)$ ] and fear of disease progression [measured using the Fear of Progression Questionnaire $(\mathrm{p}<0.01)]$. The question 2 score also correlated with younger age $(\mathrm{p}=0.01)$ and high depression scores assessed using the Patient Health Questionnaire-9 $(\mathrm{p}=0.04)$. We believe the scores for these two cancer-related dysfunctional beliefs can be used to help evaluate sleep disturbances in cancer patients.

Sleep Med Res 2017;8(2):98-101
\end{abstract}

Received: September 25, 2017

Revised: October 5, 2017

Accepted: October 11, 2017

Correspondence

Seockhoon Chung, MD, PhD

Department of Psychiatry,

Asan Medical Center, University of Ulsan

College of Medicine, 86 Olympic-ro 43-gil,

Songpa-gu, Seoul 05505, Korea

Tel +82-2-3010-3411

Fax +82-2-485-8381

E-mailschung@amc.seoul.kr

ORCID

Seockhoon Chung

https://orcid.org/0000-0002-9798-3642

Soyoung Youn

https://orcid.org/0000-0003-4983-4017

Byeongil Choi

https://orcid.org/0000-0002-9155-0598
Key Words Insomnia, Cancer, Sleep, Sleep-related cognition.

\section{INTRODUCTION}

Cancer patients frequently suffer from sleep disturbance over the course of their cancer experience trajectory. The prevalence of insomnia is higher in cancer patients than in the general population. ${ }^{1}$ Human beings commonly feel anxious and become depressed when first diagnosed with cancer. The chemotherapy, radiotherapy and hormonal therapy that ensues is often associated with sleep disturbance. ${ }^{2}$ Cancer-related physical symptoms such as pain, nausea, vomiting and hot flashes can also be reasons for sleep disturbance.

When cancer patients experience difficulty falling asleep, they often feel more anxious about consequences of their sleep disturbance than would someone free of a cancer diagnosis. In clinical practice, numerous cancer patients express concern that their disease will progress or metastasize if they cannot sleep well, and some patients worry that they will develop immune dysfunction if they cannot fall asleep by a certain, pre-determined and fixed time. And such cancer-related dysfunctional beliefs about sleep may be significantly associated with sleep disturbance. In the present study, we investigated whether cancer-related dysfunctional beliefs about sleep are associated with insomnia and other psychiatric symptoms of cancer patients. We hypothesized that cancer-related dysfunctional beliefs might be associated with the severity of the insomnia present, as well. 


\section{METHODS}

This is a retrospective medical records review study. The study protocol was approved by the Institutional Review Board of Asan Medical Center, and informed consents were not obtained from the subjects (2017-0926).

We asked ten cancer patients, who visit the Sleep Clinic for Cancer Patients of Asan Medical Center, how they regard their insomnia. The most commonly-expressed beliefs about sleep were "My immune system will have serious problems if I don't go to sleep at a certain time" (question 1, immune dysfunction) and "If I don't sleep well at night, my cancer may recur or metastasize" (question 2, cancer recurrence). Other beliefs such as "I should sleep at least eight hours a night" and "If I can't sleep, it will ruin my daily activity" were already well-known dysfunctional beliefs of primary insomnia patients. Patients were asked to rate their experience with the two beliefs on a scale $0-10$.

When cancer patients first visit the specialized Sleep Clinic for Cancer Patients at Asan Medical Center, Seoul, South Korea, their psychological states are routinely assessed using questionnaires and other rating scales such as the Insomnia Severity Index (ISI), Patient Health Questionnaire-9, Fear of Progression
Questionnaire $^{3}$ and the State anxiety items of the State-Trait Anxiety Inventory. In addition, patients' sleep structures such as sleep latency, time in bed, and time in bed during 24 hours (TIB/ d) were routinely asked. We reviewed the medical records of the subjects diagnosed with cancer, regardless of their cancer subtype, who visited the Sleep Clinic for Cancer Patients during the period January 1 through June 30, 2017, and who completed all of the rating scales on their first visit.

\section{Statistical Analysis}

Statistical analyses were performed using SPSS version 19.0 for Windows (IBM Corp., Armonk, NY, USA). Correlation and regression analyses were performed, and the level of significance was defined as $\mathrm{p}<0.05$ in two-tailed tests.

\section{RESULTS}

All of the 124 eligible cancer patients (47 men and 77 women) were included in this study and the corresponding clinical characteristics and sleep structure were presented in Table 1. The mean subjects' age was $55.1 \pm 11.2$ (range 25-84) years; the men were generally older than the women $(59.7 \pm 10.2$ vs.

Table 1. Clinical characteristics and sleep structure of the patients

\begin{tabular}{lcc}
\hline \multicolumn{1}{c}{ Variables } & Mean & Range \\
\hline Age & $55.1 \pm 11.2$ & $25-84$ \\
Time variables & & $19: 00 \mathrm{pm}-2: 30 \mathrm{am}$ \\
Bedtime & $22: 22 \mathrm{pm}$ & $21: 00 \mathrm{pm}-5: 50 \mathrm{am}$ \\
Sleep onset time & $23: 36 \mathrm{pm}$ & $3: 00 \mathrm{am}-12: 00 \mathrm{pm}$ \\
Wake up time & $6: 51 \mathrm{am}$ & \\
Duration variables & & $0-4.5 \mathrm{hr}$ \\
Sleep latency & $1.07 \mathrm{hr}$ & $4.0-13.5 \mathrm{hr}$ \\
Time in bed & $8.95 \mathrm{hr}$ & $5.0-20.0 \mathrm{hr}$ \\
Time in bed during 24 hours, TIB/d & $11.31 \mathrm{hr}$ & $1-28$ \\
Rating scales & & $0-27$ \\
Insomnia Severity Index & $16.4 \pm 6.0$ & $12-60$ \\
Patient Health Questionnaire -9 & $12.5 \pm 6.5$ & $20-62$ \\
Fear of Progression Questionnaire & $37.7 \pm 11.8$ & $41.5 \pm 8.5$ \\
State subcategory of State-Trait Anxiety Inventory & & \\
\hline
\end{tabular}

Table 2. Correlation coefficients of the two dysfunctional beliefs with age and rating scales

\begin{tabular}{|c|c|c|c|c|c|}
\hline Questions & Age & ISI & PHQ-9 & FoP-Q & STAI-S \\
\hline $\begin{array}{l}\text { Question } 1 . \text { My immune system will have serious problems if I don't } \\
\text { go to sleep at a certain time. }\end{array}$ & -0.09 & $0.32^{\dagger}$ & 0.14 & $0.34^{\dagger}$ & 0.04 \\
\hline $\begin{array}{l}\text { Question 2. If I don't sleep well at night, my cancer may recur or } \\
\text { metastasize. }\end{array}$ & $-0.22^{*}$ & $0.33^{\dagger}$ & $0.19^{*}$ & $0.40^{\dagger}$ & 0.10 \\
\hline
\end{tabular}

${ }^{*} \mathrm{p}<0.05 .{ }^{\dagger} \mathrm{p}<0.01$.

ISI: Insomnia Severity Index, PHQ-9: Patient Health Questionnaire-9, FoP-Q: Fear of Progression Questionnaire, STAI-S: state subcategory of the State-Trait Anxiety Inventory. 
$52.4 \pm 11.0$ years). Breast cancer was the most common cancer diagnosis ( $\mathrm{n}=51,30.7 \%)$, followed by lung $(\mathrm{n}=20,12.0 \%)$, pancreatic $(n=9,5.4 \%)$, and gastric cancers $(n=9,5.4 \%)$. There was no significant difference in any rating scale scores between the cancer types. The womens' scores for questions were significantly higher than the mens' (question $1: \mathrm{p}=0.04$; question $2: \mathrm{p}=$ $0.02)$. There was a significant negative correlation between age and the question 2 score $(\mathrm{r}=-0.22, \mathrm{p}=0.01)$, but not with the question 1 score.

The question 1 and question 2 scores were highly correlated with each other $(\mathrm{r}=0.64, \mathrm{p}<0.01)$. The question 1 score was significantly correlated with the severity of the insomnia $(\mathrm{r}=$ $0.32, \mathrm{p}<0.01)$ and fear of disease progression $(\mathrm{r}=0.34, \mathrm{p}<0.01)$ (Table 2). The question 2 score was significantly correlated with the severity of the insomnia $(\mathrm{r}=0.33, \mathrm{p}<0.01)$, depression $(\mathrm{r}=$ $0.19, \mathrm{p}=0.04)$ and the fear of progression $(\mathrm{r}=0.40, \mathrm{p}<0.01)$. Stepwise regression analysis revealed that the question 1 score $\left(\mathrm{R}^{2}=0.18, \mathrm{p}<0.01\right)$ was generally predicted by the fear of progression $(\beta=0.30)$ and the severity of the insomnia $(\beta=0.25)$, and that the question 2 score $\left(\mathrm{R}^{2}=0.28, \mathrm{p}<0.01\right)$ was predicted by the fear of progression $(\beta=0.34)$, the severity of the insomnia $(\beta=0.29)$ and subject's chronologic age $(\beta=-0.20)$.

\section{DISCUSSION}

In this study, we identified two cancer-related dysfunctional beliefs about sleep and showed that they were significantly correlated with the severity of cancer patients' insomnia and the degree of their fear of disease progression. Cancer patients generally suffer from insomnia, and they feel anxious about sleep disturbance. When we interviewed cancer patients attending our sleep clinic about their dysfunctional beliefs, we observed that many patients worry that their sleep disturbance could negatively impact the progression of each patient's particular cancer. These dysfunctional beliefs are rooted in valid medical knowledge; it is well known and generally accepted that sleep disturbance is associated with immune dysfunction modulated by melatonin, ${ }^{4}$ and that insufficient sleep may be linked to a significantly increased risk of cancer recurrence. ${ }^{5}$ These beliefs are designated "dysfunctional" because although the beliefs are based on valid medical information, these beliefs serve to produce anxiety in the cancer patients. This anxiety, in turn and in conjunction with the pre-existing and underlying fear of cancer recurrence, can significantly and negatively influence sleep quality $^{6}$ and in turn the immune system, and eventually becomes as a self-perpetuating, self-defeating and vicious cycle for the afflicted cancer patient.

In the present study, we found that two cancer-related dysfunctional beliefs were associated with the severity of the patients' respective insomnia conditions, and the patients' fear of disease progression. Exploring and managing dysfunctional beliefs about sleep are therapeutic tools of considerable importance when properly implemented for the purpose of reducing anxiety about sleep. The reduction of dysfunctional beliefs might play a crucial and positive role in reducing. ${ }^{7}$ However, further study is needed to determine whether changing cancer-related dysfunctional beliefs can actually be effective in diminishing sleep disturbance and reducing the insomnia of cancer patients.

In this study, the belief about cancer recurrence (question 2) was found to be associated with depression and a younger age, but the belief about immune dysfunction (question 1) was not. The thought of "cancer recurrence" is an issue of significant and ongoing fear and anxiety for cancer patients in general, ${ }^{8}$ and cancer patients diagnosed at a relatively young age, in particular. ${ }^{9}$ In contrast, anxiety measured by the State-Trait Anxiety Inventory does not concern itself with either of the specific dysfunctional beliefs (or the related ramifications) investigated by this study. In view of the foregoing, we can only speculate and surmise that the potentially formidable fear of "disease progression" has greater impact on cancer-related dysfunctional beliefs than the aggregate of any and all other symptoms of general anxiety that might be present otherwise.

Limitations of this study include use of the ISI, a simple rating scale, to assess patients' insomnia symptoms. With more objective sleep data, we could have explored the association between cancer-related dysfunctional beliefs and objective sleep indices.

We acknowledge another constraint: We lacked the wherewithal to limit the qualified subjects to patients with specific cancer diagnoses. We believe it both advisable and necessary that we further explore the potential connection between dysfunctional beliefs and insomnia as potentially influenced by the particular type of cancer afflicting the particular patient. Lastly, we could not make adjustments in the study protocol to accommodate the effect of the actual stage of the patient's diagnosed cancer, or the impact of factors such as fatigue or level and/or chronicity of physical pain. Certainly, it is possible that the aforementioned elements, and other factors unaccounted for, could also serve to substantially influence the patients' sleep disturbances based on a chronic and underlying fear of cancer progression.

In conclusion, we identified two cancer-related specific dysfunctional beliefs not infrequently held by cancer patients, and we found that those beliefs were directly related to both the severity of the cancer patients' insomnia, and the cancer patients' fear of their particular cancer advancing and/or metastisizing.

\section{Conflicts of Interest}

The authors have no financial conflicts of interest.

\section{Authors' Contribution}

Conceptualization: Chung S. Data curation: Chung S, Youn S, Choi B. Formal analysis: Chung S. Investigation: Chung S, Youn S, Choi B. Methodology: Chung S, Youn S. Project administration: Chung S. Resources: Chung S, Youn S, Choi B. Software: Chung S. Supervision: Chung S. Val- 
idation: Chung S, Youn S, Choi B. Writing-original draft: Chung S. Writing_review \& editing: Chung S, Youn S, Choi B.

\section{REFERENCES}

1. Park B, Youn S, Hann CC, Yi K, Lee S, Lee JS, et al. Prevalence of insomnia among patients with the ten most common cancers in South Korea: Health Insurance Review and Assessment Service-National Patient Sample. Sleep Med Res 2016;7:48-54.

2. Ancoli-Israel S. Sleep disturbances in cancer: a review. Sleep Med Res 2015;6:45-9.

3. Shim EJ, Shin YW, Oh DY, Hahm BJ. Increased fear of progression in cancer patients with recurrence. Gen Hosp Psychiatry 2010;32:169-75.

4. Haus EL, Smolensky MH. Shift work and cancer risk: potential mechanistic roles of circadian disruption, light at night, and sleep deprivation. Sleep Med Rev 2013;17:273-84.

5. Thompson CL, Li L. Association of sleep duration and breast cancer
OncotypeDX recurrence score. Breast Cancer Res Treat 2012;134:1291-5.

6. Berrett-Abebe J, Cadet T, Pirl W, Lennes I. Exploring the relationship between fear of cancer recurrence and sleep quality in cancer survivors. J Psychosoc Oncol 2015;33:297-309.

7. Eidelman P, Talbot L, Ivers H, Bélanger L, Morin CM, Harvey AG. Change in dysfunctional beliefs about sleep in behavior therapy, cognitive therapy, and cognitive-behavioral therapy for insomnia. Behav Ther 2016;47:102-15.

8. Koch L, Bertram H, Eberle A, Holleczek B, Schmid-Höpfner S, Waldma$\mathrm{nn} \mathrm{A}$, et al. Fear of recurrence in long-term breast cancer survivors-still an issue. Results on prevalence, determinants, and the association with quality of life and depression from the cancer survivorship--a multi-regional population-based study. Psychooncology 2014;23:547-54.

9. Lebel S, Beattie S, Arès I, Bielajew C. Young and worried: age and fear of recurrence in breast cancer survivors. Health Psychol 2013;32:695705. 